\title{
Paroxysmal Autonomic Instability With Dystonia Managed Using Chemodenervation Including Alcohol Neurolysis and Botulinum Toxin Type A Injection: A Case Report
}

Hye-Sun Lee, MD, Hyun-Seung Oh, MD, Joon-Ho Shin, MD, MS

Department of Rehabilitation Medicine, National Rehabilitation Center, Seoul, Korea

Paroxysmal autonomic instability with dystonia (PAID) is a rare complication of brain injury. Symptoms of PAID include diaphoresis, hyperthermia, hypertension, tachycardia, and tachypnea accompanied by hypertonic movement. Herein, we present the case of a 44 -year-old female patient, who was diagnosed with paraneoplastic limbic encephalopathy caused by thyroid papillary cancer. The patient exhibited all the symptoms of PAID. On the basis that the symptoms were unresponsive to antispastic medication and her liver function test was elevated, we performed alcohol neurolysis of the musculocutaneous nerve followed by botulinum toxin type A (BNT-A) injection into the biceps brachii and brachialis. Unstable vital signs and hypertonia were relieved after chemodenervation. Accordingly, alcohol neurolysis and BNT-A injection are proposed as a treatment option for intractable PAID.

Keywords Paroxysmal autonomic instability with dystonia, Botulinum toxins, Nerve block, Dystonia, Autonomic nervous system

\section{INTRODUCTION}

Paroxysmal autonomic instability with dystonia (PAID) is a rare clinical manifestation that appears subsequent

Received May 13, 2014; Accepted August 4, 2014

Corresponding author: Joon-Ho Shin

Department of Rehabilitation Medicine, National Rehabilitation Center, 58 Samgaksan-ro, Gangbuk-gu, Seoul 142-884, Korea

Tel: +82-2-901-1884, Fax: +82-2-901-1899, E-mail: asfreelyas@gmail.com

(c) This is an open-access article distributed under the terms of the Creative Commons Attribution Non-Commercial License (http://creativecommons. org/licenses/by-nc/3.0) which permits unrestricted noncommercial use, distribution, and reproduction in any medium, provided the original work is properly cited.

Copyright ( 2015 by Korean Academy of Rehabilitation Medicine to central nervous system injuries, regardless of the underlying etiology $[1,2]$. Symptoms of PAID include hyperthermia, hypertension, tachycardia, tachypnea, intermittent agitation, and diaphoresis accompanied by hypertonia [1]. Despite the development of various pharmacological treatment options, some cases of PAID still remain intractable. In this study, we present the case of a 44-year-old female patient with paraneoplastic limbic encephalitis that was accompanied by intractable symptoms related to PAID. We propose that the use of chemodenervation to control hypertonia may be a viable and novel treatment option for intractable PAID. 


\section{CASE REPORT}

A female patient was diagnosed with paraneoplastic limbic encephalitis originating from thyroid cancer in January 2013 and was admitted to our hospital 4 months after diagnosis. At the time of admission, she was bedridden and presented severe tonic posturing tendency of both elbow and wrist flexions as well as ankle plantar flexion (Fig. 1A). She was on a medication regimen of carbidopa/levodopa (150/600 mg/day), amantadine (400 $\mathrm{mg} /$ day), baclofen (90 mg/day), diazepam (100 mg/day), eperisone (200 mg/day), bisoprolol ( $2.5 \mathrm{mg} /$ day), ranitidine (300 mg/day), and synthyroxine $(0.15 \mathrm{mg} /$ day).

One week after admission, eperisone administration (200 mg/day) was discontinued and baclofen (90 mg/ day) was tapered down slowly (to $60 \mathrm{mg} /$ day), because the results of liver function test suggested that the current regimen was toxic (aspartate aminotransferase [AST], $88 \mathrm{IU} / \mathrm{L}$; alanine aminotransferase [ALT], $69 \mathrm{IU} / \mathrm{L}$; $\gamma$-glutamyltranspeptidase [ $\gamma \mathrm{GTP}], 142 \mathrm{IU} / \mathrm{L})$. Subsequently, sudden dystonic movements, such as grimace, neck pulling, further aggravated wrist flexion, trunk bending, leg extension, and ankle plantar flexion with severe elbow flexion, appeared. At the same time, diaphoresis, fever (up to $39.4^{\circ} \mathrm{C}$ ), elevated blood pressure (up to $168 / 85$ $\mathrm{mmHg}$ ), tachycardia (up to $118 / \mathrm{min}$ ), and tachypnea (up to $38 / \mathrm{min}$ ) appeared in multiple episodes. To mitigate sudden dystonic movements and unstable vital signs, we suggested bed rest and position changes accompanied with emergency care. This procedure led to improvement in dystonic movement; however, severe dystonic elbow flexor posture and unstable vital signs continued. Intramuscular lorazepam ( $2 \mathrm{mg}$ or $4 \mathrm{mg}$ ) was frequently administered during the episodes, thus stabilizing her tonic movements and vital signs.

However, after a few days, the symptoms were aggravated. The body temperature increased to $39.4^{\circ} \mathrm{C}$ accompanied by tachycardia (up to $120 / \mathrm{min}$ ) and tachypnea (up to $32 / \mathrm{min}$ ). Pulse oximetry showed $88 \%$ saturation of oxygen, and $2 \mathrm{~L}$ of oxygen was administered. Blood pressure was not recorded during this episode because of severe tonic movements. In addition, no response of dystonic movement to intramuscular lorazepam injection was noted. Laboratory results revealed leukocytosis $(12,400 / \mathrm{mL}$ with neutrophilia at $81 \%$ of the differential count), and pyuria (white blood cells, 50-99/high-power field). This episode was assumed as a sign of infection; hence, urine bacterial culture was performed. Culture results showed the growth of extended-spectrum betalactamase-positive bacteria, accordingly imipenem (1,000 $\mathrm{mg} /$ day) was administered. Despite improved laboratory test results after 5 days of imipenem administration, fever persisted in a periodic manner with other consistently unstable vital signs. Therefore, the diagnosis of infection was revoked and we turned our attention to other potential causes. Additional tests including electroencephalography, brain computed tomography, and spinal fluid tapping were performed. All results showed inconclusive findings. During this period, baclofen dosage was increased (up to $90 \mathrm{mg} /$ day) and eperisone was
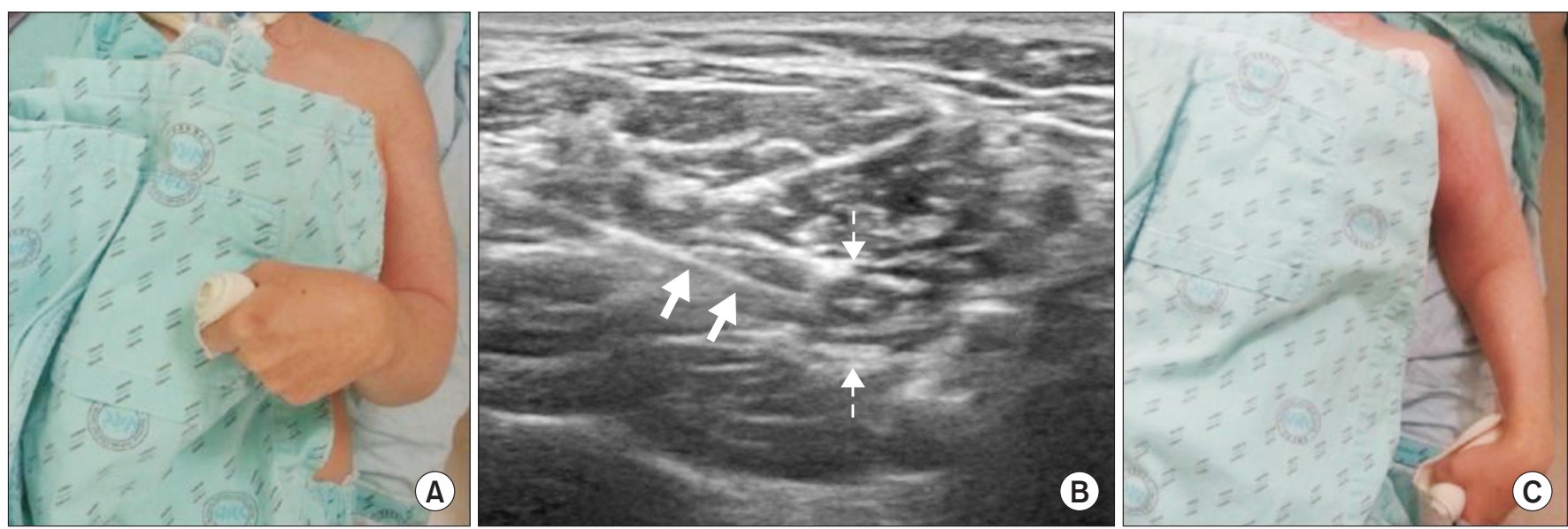

Fig. 1. (A) Initial elbow flexed posture. (B) Ultrasound-guided musculocutaneous neurolysis with alcohol (solid arrow, needle; dashed arrow, musculocutaneous nerve). (C) Extended elbow posture after alcohol neurolysis. 
again administered (up to $200 \mathrm{mg}$ /day). Subsequently, stabilization in vital signs was noted with disappearance of myoclonic movements, and decreased hypertonia.

One month after the last episode, a similar event occurred and empirical antibiotics were administered, but the outcomes were not positive. We hypothesized that sustained contracting muscle could be the cause of fever. Thus, instead of antibiotics, dantrolene was administered, which produced positive results. However, later, administration of dantrolene was discontinued because of elevated AST and ALT levels. Consequently, we performed $50 \%$ alcohol neurolysis on her left musculocutaneous nerve under ultrasonography guidance (Accuvix XG; Medison, Seoul, Korea) (Fig. 1B). After the procedure, sustained contraction of her left elbow was immediately relieved (Fig. 1C), and stabilization of clinical vital signs was noted. Occasional episodes of fever, tachypnea, and hypertension were observed, but with decreased frequency (Fig. 2).

Again, a single episode of severe fever with increased dystonia was observed 7 days after the left musculocutaneous neurolysis. At this point, we attempted botulinum
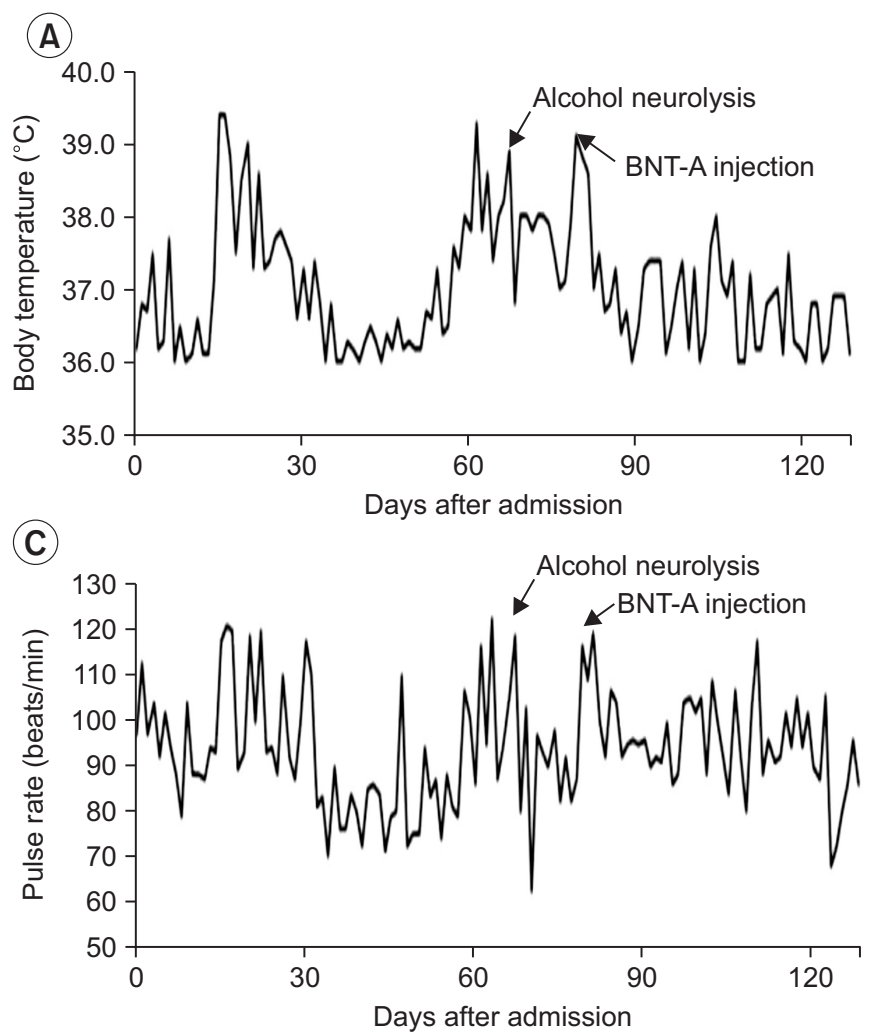

toxin type A (BNT-X) (Botox; Allergan Inc., Irvine, CA, USA) injection into the right biceps brachii (40 units) and brachialis (60 units). In less than a week's time after BNTA injection, hypertonia and unstable vital signs were relieved. During the subsequent 8 months of follow-up, no episodes of increased body temperature and blood pressure, tachycardia, and tachypnea were presented, even though right elbow flexor muscle contraction resumed. The change in the Global Dystonia Rating Scale [3] is shown in Table 1.

Nine months after the last chemodenervation, aggravation of right elbow flexor contraction along with initiation of symptoms was noted again. Right musculocutaneous neurolysis with $50 \%$ alcohol was administered and the symptoms disappeared.

\section{DISCUSSION}

PAID is a complication of severe brain injury, such as traumatic brain injury, brain anoxia, subarachnoid and intracranial hemorrhages, midbrain glioma, and hydrocephalus $[1,2]$. Clinical manifestations of PAID include
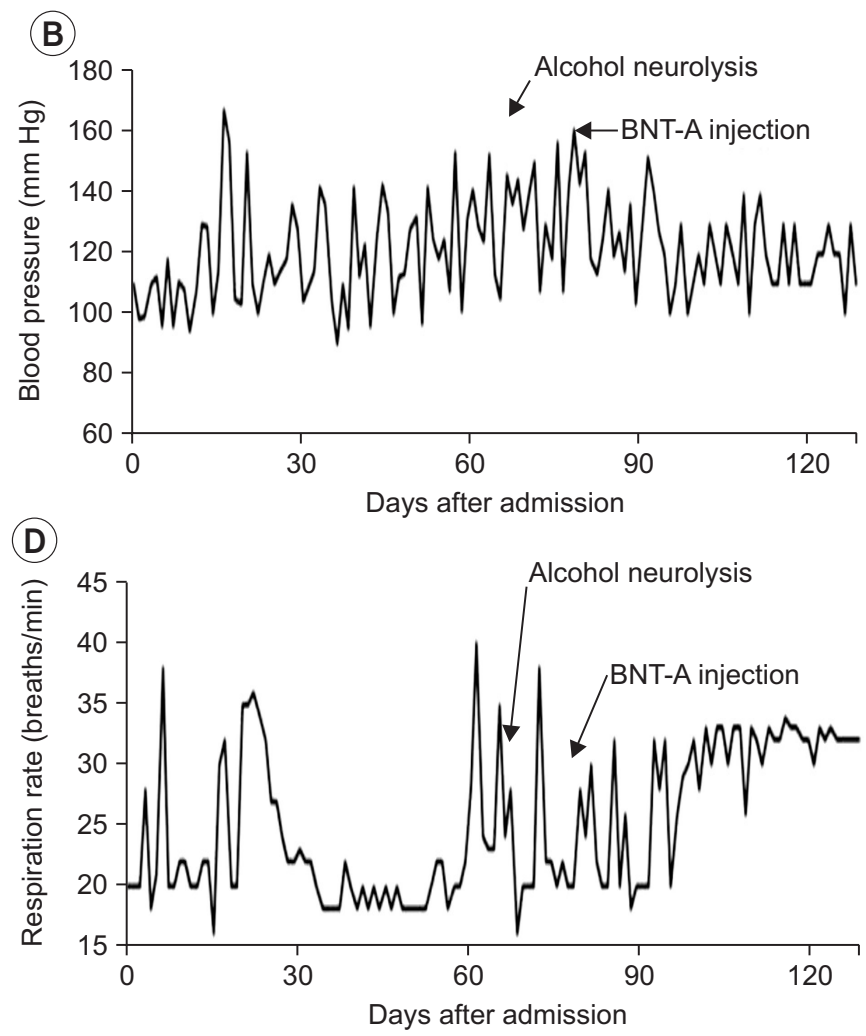

Fig. 2. Change in vital signs with alcohol neurolysis and botulinum toxin type A (BTN-A) injection. (A) Body temperature, (B) systolic blood pressure, (C) pulse rate, and (D) respiration rate. 


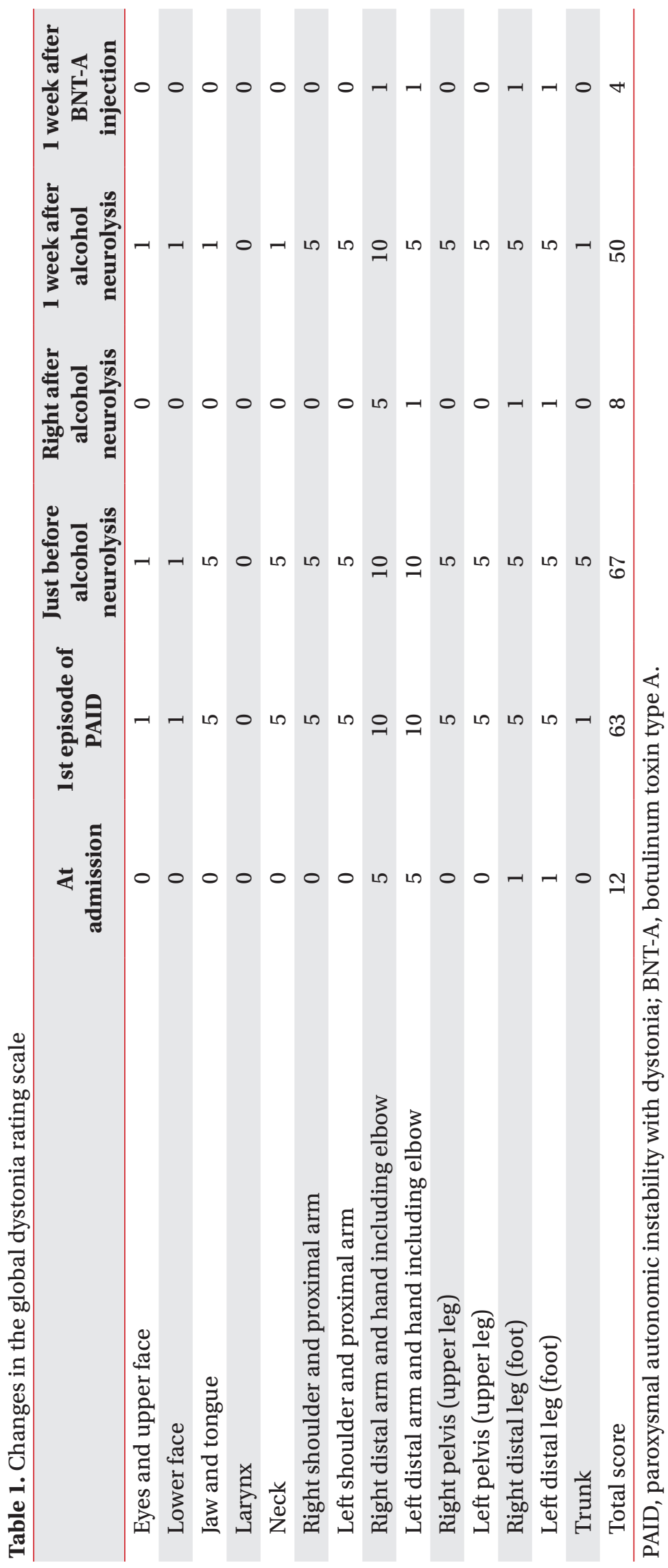

intermittent agitation, diaphoresis, hyperthermia, hypertension, tachycardia, and tachypnea accompanied by hypertonia [1]. All the symptoms of PAID were manifested in our case. Fever manifestation was the main clinical feature that led to misdiagnosis in the present case. Blood laboratory tests and imaging were performed to investigate the cause of fever and the results suggested an infection, such as a urinary tract infection or pneumonia as probably the major cause. The acute nature of the patient's symptoms supported this hypothesis. Thus, antibiotics were tried as a first line of treatment, but they showed little effect. Other evaluation methods also failed to determine the cause of fever. It was further observed that abnormal autonomic symptoms did not precede hypertonia in multiple episodes. Therefore, it was concluded that the patient's symptoms did not result from infection, but likely from sustained muscle contraction. Dysautonomia in PAID was related to triggering events, such as stimulation of muscle mechanoreceptor [4]; thus, the dystonia observed in this study might have caused the dysautonomia. In addition, sustained muscle contraction was regarded as a cause of hyperpyrexia [5].

Previous research has suggested many possible treatment options for PAID, including administration of morphine sulfate, bromocriptine mesylate, propranolol hydrochloride, clonidine hydrochloride, benzodiazepine, dantrolene sodium, baclofen infusion, and dexmedetomidine [4,6-9]. Baclofen, diazepam, and dantrolene were administered in the present case, but their effect was limited only to quite a few days. Increasing the dosages of the medications was not an option, because the patient's liver function test showed increased levels of AST and ALT. Therefore, alcohol neurolysis and intramuscular BNT-A injection, which had never been previously reported in PAID, were attempted instead. We designed this treatment plan based on two lines of reasoning. First, chemodenervation can relieve excessive muscle contraction accompanying hypermetabolism. Generally, alcohol splits myelin sheaths, and 
subsequently causes neuronal degeneration [10], thus causing transient paralysis. BNT-A interrupts acetylcholine release into the synaptic cleft, thus temporarily disabling the neuromuscular junction. Second, unlike oral medications, the effects of alcohol neurolysis and BNTA injection are not systemic but localized; thus, they are unlikely to lead to unwanted systemic side effects. In order to confirm that the improvement in PAID symptoms was not due to alcohol itself but due to muscle relaxation or dystonia relief, we applied BNT-A instead of alcohol during the following PAID episode.

In addition, it was observed that the patient's PAID symptoms correlated with the extent of muscle contraction. When contraction of the left elbow flexor was relieved, the PAID symptoms subsided, but were not completely resolved. Subsequently, when control of the right elbow flexor was attained, the effect was sufficient to resolve the PAID symptoms. As the effects of BNT-A decreased, right elbow flexor contraction, fever, hypertension, tachypnea and diaphoresis resumed. An additional chemoneurolysis injection (9 months after the last chemodenervation) relieved muscle contraction and eventually relieved PAID symptoms. Both, alcohol neurolysis and BNT-A injection relaxed muscle contraction and relieved the symptoms of PAID. Thus, we propose that alcohol neurolysis and BNT-A injection can be considered a treatment option for intractable PAID.

\section{CONFLICT OF INTEREST}

No potential conflict of interest relevant to this article was reported.

\section{ACKNOWLEDGMENTS}

This study was supported by a grant from the Technology Innovation Program (No. 10046150) funded by the Ministry of Trade, Industry \& Energy (Korea).

\section{REFERENCES}

1. Blackman JA, Patrick PD, Buck ML, Rust RS Jr. Paroxysmal autonomic instability with dystonia after brain injury. Arch Neurol 2004;61:321-8.

2. Ramdhani NA, Sikma MA, Witkamp TD, Slooter AJ, de Lange DW. Paroxysmal autonomic instability with dystonia in a patient with tuberculous meningitis: a case report. J Med Case Rep 2010;4:304.

3. Comella CL, Leurgans S, Wuu J, Stebbins GT, Chmura T; Dystonia Study Group. Rating scales for dystonia: a multicenter assessment. Mov Disord 2003;18:303-12.

4. Boeve BF, Wijdicks EF, Benarroch EE, Schmidt KD. Paroxysmal sympathetic storms ("diencephalic seizures") after severe diffuse axonal head injury. Mayo Clin Proc 1998;73:148-52.

5. Sneed RC. Hyperpyrexia associated with sustained muscle contractions: an alternative diagnosis to central fever. Arch Phys Med Rehabil 1995;76:101-3.

6. Goh KY, Conway EJ, DaRosso RC, Muszynski CA, Epstein FJ. Sympathetic storms in a child with a midbrain glioma: a variant of diencephalic seizures. Pediatr Neurol 1999;21:742-4.

7. Thorley RR, Wertsch JJ, Klingbeil GE. Acute hypothalamic instability in traumatic brain injury: a case report. Arch Phys Med Rehabil 2001;82:246-9.

8. Cuny E, Richer E, Castel JP. Dysautonomia syndrome in the acute recovery phase after traumatic brain injury: relief with intrathecal Baclofen therapy. Brain Inj 2001;15:917-25.

9. Goddeau RP Jr, Silverman SB, Sims JR. Dexmedetomidine for the treatment of paroxysmal autonomic instability with dystonia. Neurocrit Care 2007;7:217-20.

10. Taylor JJ, Woolsey RM. Dilute ethyl alcohol: effect on the sciatic nerve of the mouse. Arch Phys Med Rehabil 1976;57:233-7. 